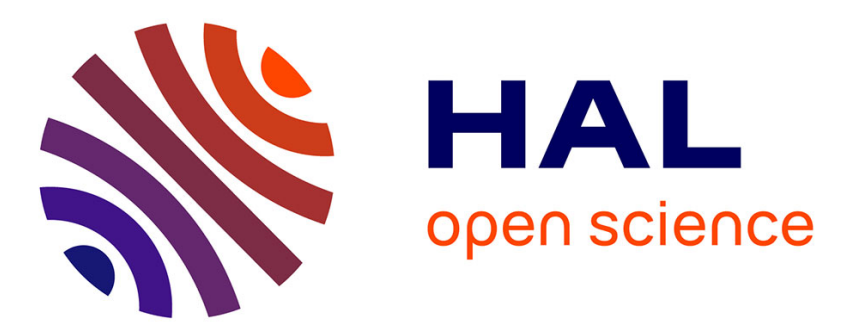

\title{
Observer and model predictive control for on-line parameter identification in nonlinear systems
}

\author{
Jun Qian, Pascal Dufour, Madiha Nadri
}

\section{To cite this version:}

Jun Qian, Pascal Dufour, Madiha Nadri. Observer and model predictive control for on-line parameter identification in nonlinear systems. IFAC International Symposium on Dynamics and Control of Process Systems (DYCOPS), Dec 2013, Mumbai, India. pp.571-576, 10.3182/20131218-3-IN-2045.00042 . hal-01025666

\section{HAL Id: hal-01025666 https://hal.science/hal-01025666}

Submitted on 18 Jul 2014

HAL is a multi-disciplinary open access archive for the deposit and dissemination of scientific research documents, whether they are published or not. The documents may come from teaching and research institutions in France or abroad, or from public or private research centers.
L'archive ouverte pluridisciplinaire HAL, est destinée au dépôt et à la diffusion de documents scientifiques de niveau recherche, publiés ou non, émanant des établissements d'enseignement et de recherche français ou étrangers, des laboratoires publics ou privés. 
This document must be cited according to its final version which is published in a conference proceeding as:

J. Qian ${ }^{123}$, P. Dufour ${ }^{1}$, M. Nadri ${ }^{1}$
Observer and model predictive control for on-line parameter
identification in nonlinear systems"',
Proceedings of the 10th IFAC International Symposium
on Dynamics and Control of Process Systems (DYCOPS),
Mumbai, India, pp. 571-576, 2013.

All open archive documents of Pascal Dufour are available at: http://hal.archives-ouvertes.fr/DUFOUR-PASCAL-C-3926-2008

The professional web page (Fr/En) of Pascal Dufour is: http://www.lagep.univ-Iyon1.fr/signatures/dufour.pascal

The web page of this research group is: http://hal.archives-ouvertes.fr/SNLEP

1

Université de Lyon, Lyon, F-69003, France; Université Lyon 1;

CNRS UMR 5007 LAGEP (Laboratoire d'Automatique et de GEnie des Procédés),

43 bd du 11 novembre, 69100 Villeurbanne, France

Tel +33 (0) $472431845-$ Fax +33 (0) 472431699

http://www-lagep.univ-lyon1.fr/ http://www.univ-lyon1.fr http://www.cnrs.fr

2

Acsysteme

Company Acsystème, 4 rue Ren é Dumont, 35000 Rennes, France.

Emails: jun.qian@acsysteme.com

3 This PhD thesis is between the LAGEP and the french company Acsystème which is gratefully acknowledged for the funding. The french ministry of higher education and research, for the financial support of this CIFRE PhD thesis 2011/0876, is also acknowledged. 


\title{
Observer and model predictive control for on-line parameter identification in nonlinear systems *
}

\author{
Jun Qian ${ }^{*, * *}$ Pascal Dufour ${ }^{* *, * * *}$ Madiha Nadri ${ }^{* *}$ \\ * Acsystème, immeuble Cap nord A, 2 Allée Marie Berhaut, \\ 35000 Rennes, France (e-mail: jun.qian@acsysteme.com) \\ ** Université de Lyon, Lyon F-69003, Université Lyon 1, CNRS UMR \\ 5007, Laboratory of Process Control and Chemical Engineering \\ (LAGEP), Villeurbanne 69100, France (e-mail: \\ qian@lagep.univ-lyon1.fr; dufour@lagep.univ-lyon1.fr; \\ nadri@lagep.univ-lyon1.fr) \\ *** Corresponding author.
}

\begin{abstract}
This paper develops an on-line model parameter identification approach for multivariable systems, which are nonlinear in terms of state representation and/or in terms of parameters. Combining the observation theory and the model based predictive control theory, an optimal closed loop experiment design for on-line identification of model parameters is given. During only one experiment, an optimal time-varying input is computed to optimize a criterion, while the unknown model parameters are estimated at the same time. The criterion is based on the sensitivities of the model outputs with respect to the unknown parameters that are estimated. The approach does not require to measure all the process state. Moreover output constraints allow to maintain the behaviour into a prescribed region and/or stabilize the process in closed loop. This approach is illustrated through an unstable rolling delta wing with one input, two measured states and five unknown constant model parameters.
\end{abstract}

Keywords: Optimal experiment design, identification, nonlinear systems, observers, predictive control.

\section{INTRODUCTION}

The increasing use of numerical techniques leads to consider more complex and more realistic models in process modelling, where all model parameters need to be numerically known. Therefore, since the 1970's, optimal experiment design (OED) is an area of considerable interest for the purpose of identification (see Mehra [1974], Goodwin and Payne [1979], Zarrop [1979] and the recent review paper of Franceschini and Macchietto [2008]). In OED, most of the literature has focused on system approximated by a linear model, whereas in many areas such as biological and chemical processes, the models are highly nonlinear. Moreover, in classical identification techniques, several experiments are usually required. Hence, it does affect the duration of using of the process for pure identification and the cost of these particular experiments (for example, material fed at the process inlet, energy consumption during the experiment, output materials with undesired properties).

Furthermore, model parameters are often estimated after running new (or collecting past) experiments (Goodwin and Payne [1979]). These data are then usually fed into a Fisher Information Matrix (FIM) which is manipulated in

* This $\mathrm{PhD}$ thesis is between the LAGEP and the french company Acsystème which is gratefully acknowledged for the funding. The french ministry of higher education and research, for the financial support of this CIFRE PhD thesis 2011/0876, is also acknowledged. a estimation algorithm based on an optimal cost function. Hence, one drawback is that these data may not contain enough information to estimate all parameters. Also, proof of convergence of the estimator has to be given.

Recently, Biao Huang and his colleagues developed online OED techniques with combined parameter estimation in the multivariable case but without output constraint (Jayasankar et al. [2010]). These authors added linear equality constraints obtained from the steady state analysis in a constrained extended Kalman filter (Zhu and Huang [2011]). These developed techniques for closed-loop control for identification purpose were addressed to open loop stable systems.During such experiment, it is often also of interest to account for process constraints (dealing with safety, maintain process state within bounds, production, economic performance, user comfort, ...). Therefore, a closed loop constrained optimal control approach must be used. Model Predictive Control (MPC) strategy is widely used for real-time optimal constrained control in industrial applications. Over the receding horizon, such a controller solves on-line an optimization problem, which is based on a cost function and constraints on manipulated inputs, estimated states and outputs.

Based on MPC and on nonlinear observer theory, an approach of closed-loop optimal experiment design for online identification was proposed in Flila et al. [2008]. In this work, authors considered the mono-variable case (a single input, a single measured state and a single unknown 
constant parameter) for stable nonlinear systems.

The present paper investigates some issues with the constrained closed loop optimal design of one experiment used simultaneously for on-line parameter identification of the unknown nonlinear model parameters (the non-linearity is in terms of dynamics and/or in terms of model parameters). The coupled observer-MPC approach initially developed in Flila et al. [2008] is extended to a general case of nonlinear systems. Contrarily to this previous work, the state may here not be entirely measured and the unknown parameters to estimate may be time-varying. Moreover input and also output constraints are specified, such that this controller may be applied to an open loop stable or unstable, linear or nonlinear, multivariable system.

Based on the chosen model structure, an observer is designed for the augmented system resulting in a combined on-line estimation of the unmeasured states and the unknown parameters. Then, a sensitivity model is used to get the sensitivity matrices of the states and the outputs of the model with respect to the unknown model parameters. Finally, the OED problem is formulated in a MPC strategy: the optimization aims to maximize on-line, over a receding horizon, the sensitivities of the model outputs with respect to the unknown model parameters under input and output constraints. The outputs of the process, the model, the observer, and the sensitivity model are used by the control law. The optimal inputs and the parameter estimations are both determined on-line.

This paper is structured as follows. Section 2 deals with the existing requirements and results needed in the section 3 , where the proposed approach of constrained closed-loop optimal design of one experiment for on-line identification is presented. The different steps of the implementation of this approach are illustrated in section 4 on a rolling delta wing, which is a nonlinear unstable system, with one input, 2 states and 5 unknown constant model parameters to determine. Hence, time-varying inputs applied during the experiment have to be designed to both stabilize the process and to obtain rich data for on-line model parameter identification. The obtained simulation results for this case study are given and discussed.

\section{PRELIMINARIES}

\subsection{Process considered}

This approach is dedicated to processes that feature some dynamic behaviour. Meanwhile, at least one on-line measure $y_{p}(t)$ must be available in output and at least one exogenous input $u(t)$ must be manipulated on-line by a controller. Some constraints may be specified on the magnitude and velocity of the manipulated input. Other constraints may be specified on the measured outputs or estimation of the process states (dealing with safety, stability, set-point tracking within bounds, production, economic performance, user comfort, ...). Hence, this covers a very large number of potential applications.

\subsection{Class of models considered}

A large class of industrial processes can be modelled by a linear or nonlinear dynamic system. The non-linearity is viewed in terms of state representation and/or in terms of model parameters. All over the paper the model under consideration will be described by a general state-space representation of the following form

$$
(M)\left\{\begin{array}{l}
\dot{x}(t)=f(x(t), \theta, u(t)) \\
y(t)=h(x(t), \theta, u(t)),
\end{array}\right.
$$

where $x \in \mathcal{R}^{n}$ is the state vector, $y$ is the vector of measured outputs taking values in some open subset of $\mathcal{R}^{n}, u$ denotes the vector of known manipulated inputs taking values in some open subset $\mathcal{U} \subset \mathcal{R}^{m}, \theta \in \mathcal{R}^{q}$ is the unknown model parameters vector (which are all constant in this study), $f$ and $h$ are nonlinear functions of suitable dimensions which will be assumed to be $C^{\infty}$ w.r.t. their arguments.

\subsection{Standard observer design}

In terms of parameter identification, observer techniques are interesting tools to estimate on-line unmeasured states and unknown model parameters for nonlinear systems. It can be an high gain observer, an extended Kalman filter or an adaptive-gain observer (see Besançon [2007], Boizot et al. [2010], Zhou et al. [2009], Nadri et al. [2004, 2013] ). The choice of the observer type depends on the model structure, the observability conditions and the persistency of excitation. Based on the model (1) and on the available on-line measures of inputs $u(t)$ and outputs $y_{p}(t)$, an observer is constructed. It gives an estimation of the process state $x(t)$ and the process parameters $\theta$. The observer design is based on the augmented model given by

$$
\left(M_{a}\right)\left\{\begin{array}{l}
\dot{x}(t)=f(x(t), \theta, u(t)) \\
\dot{\theta}=0 \\
y(t)=h(x(t), \theta, u(t)),
\end{array}\right.
$$

In the follows, we denote the augmented state vector by $x_{a}=\left[\begin{array}{ll}x & \theta\end{array}\right]^{T}$ and $f_{a}=\left[\begin{array}{ll}f & 0\end{array}\right]^{T}$.

Definition 1. : A global observer for system (2) can be given by a dynamical system on the form:

$$
(O)\left\{\begin{aligned}
\dot{\hat{x}}_{a}(t) \quad=f_{a}\left(\hat{x}_{a}(t), u(t)\right)+\ldots \\
\\
\ldots g_{a}\left(t, h\left(\hat{x}_{a}(t), u(t)\right)-y_{p}(t)\right) \\
\text { with: } \quad g_{a}(t, 0)=0
\end{aligned}\right.
$$

such that

i) if $\hat{x}_{a}(0)=x_{a}(0)$, then $\hat{x}_{a}(t)=x_{a}(t), \quad \forall t \geq 0$;

ii) if $\forall x_{a}(0), \forall \hat{x}_{a}(0)$, then $\lim _{t \rightarrow+\infty}\left\|\hat{x}_{a}(t)-x_{a}(t)\right\|=0$, where $g_{a}$ is a function of the output estimation error to be designed and $y_{p}$ is the process output vector (real measures).

Our estimation problem consists in determining a gain $g_{a}$ such that the estimation error $e(t)=x_{a}(t)-\hat{x}_{a}(t)$ converges asymptotically to zero.

\subsection{MPC techniques}

MPC is widely used in the industry (Richalet and O'Donovan [2009]) since the first implementations in the 1970's. The idea of the MPC strategy is to solve on-line, at each time $k$, a constrained optimization problem based on a criterion $J$. The controller aims to determine a sequence of inputs over the prediction horizon $N_{p}$, at the current time $k$ (e.g. $\left.\left\{u(k), u(k+1), \ldots, u\left(k+N_{p}\right)\right\}\right)^{1}$. The first

\footnotetext{
1 To simplify the notation, here, $s(k)$ (resp. $s(l)$ ) represents the value of the signal $s$ at the current (resp. future) discrete time $k \times T_{s}$
} 
component of this control sequence is then applied at the next sampling time $k+1$ where the new measurement $y_{p}(k+1)$ will be used to update the current estimation from $\hat{x}_{a}(k)$ to $\hat{x}_{a}(k+1)$. The procedure is then iterated. In terms of optimal control, the classical MPC framework aims to drive the process state to a initial target desired value (i.e. set-point or trajectory tracking). Since a few years, economic MPC (Rawlings et al. [2012]) aims to maintain the process state into a particular steady-state or time-varying state (that is initially unknown), but which optimizes the defined economic cost function while keeping process operations in a specified zone (defined by output constraints) like in building heating, ventilating, and air conditioning (Ma et al. [2012]) or chemical reactors (Heidarinejad et al. [2013]). With some kind of economic cost function based on the Fisher Information Matrix, MPC has also been used in on-line input design for identification purpose in the linear case in Genceli and Nikolaou [1996] and since a few years in the nonlinear case (Flila et al. [2008], Jayasankar et al. [2010], Zhu and Huang [2011]). In these cases, the cost function is derived from a sensitivity model.

Techniques for closed-loop identification of unstable systems exist, but mostly in the linear case (e.g. Forssell and Ljung [1998]). In nonlinear MPC, instability is often tackled in terms of control of the process as close as possible to an open-loop unstable steady state (which is of production interest), like in (Biagiola and Figueroa [2004]). In Heidarinejad et al. [2013], a Lyapunov based approach is used in an economic MPC with a two-mode operation controller that helps to stabilize the system state in closed loop in a prescribed relatively large region.

\subsection{Sensitivity model}

As we can see in the previous section, the measurement of the complete state $x(t)$ is not necessary for the development of the proposed approach. Indeed, it is sufficient to measure a combination of some states which allows to design an observer for the estimation of the unknown states and unknown parameters. However, in this case, the development of the sensitivity matrix is very fastidious and reduces the paper readability. So to better illustrate the different steps, and without loss of generality, we can now assume that the whole state is measured.

Using the definition of the sensitivity function $(\cdot)_{\theta}=\frac{\partial(\cdot)}{\partial \theta}$ of a variable $(\cdot)$ with respect to the parameters $\theta$, and the dynamical model $(M)$, we give the sensitivity model as follows

$$
\left(M_{\theta}\right)\left\{\begin{array}{l}
\dot{x}_{\theta}(t)=\frac{\partial f(x(t), \theta, u(t))}{\partial x} x_{\theta}+\frac{\partial f(x(t), \theta, u(t))}{\partial \theta} \\
y_{\theta}(t)=x_{\theta}(t),
\end{array}\right.
$$

where $x_{\theta} \in R^{n \times q}$ and $y_{\theta} \in R^{n \times q}$ are the matrices of sensitivities of the states (the outputs) with respect to the parameters. Therefore, the resolution of the dynamic

(resp. $l \times T_{s}$ ), where $T_{s}$ is the constant sampling time. For the input, a zero order hold is used between two consecutive sampling times. The various models are still formulated in a continuous framework and are solved numerically. Hence, sampled values may be taken at any discrete time. The initial value of the model state at $k$ is obtained from the real measurements or from the state estimated by the observer. It is assumed that process data may also be sampled at the same rate. model $\left(M_{\theta}\right)$ is coupled to the resolution of the dynamic model $(M)$. Physical values involved in the sensitivities have usually different scales and units. So, in order to rescale the effects of different parameters on the different outputs of the model, each sensitivity is normalized with the relative-sensitivity function

$$
\left\{\begin{array}{l}
\bar{x}_{\theta}(i, j)=\frac{\theta_{j}}{x_{i}} x_{\theta}(i, j) ; \quad i=1, \cdots, n ; j=1, \cdots, q \\
\bar{y}_{\theta}(i, j)=\frac{\theta_{j}}{y_{i}} y_{\theta}(i, j) ; \quad i=1, \cdots, r ; j=1, \cdots, q .
\end{array}\right.
$$

\section{PROPOSED APPROACH}

The approach aims to optimally design on-line, under some specified constraints, one experiment (i.e. to compute online the time-varying inputs to apply). The input/output process data are fed on-line into an observer to estimate also on-line the model parameter values and the unmeasured states. MPC is used to get the optimal control, where particular output constraints can be specified to stabilize the system (if it is open-loop unstable) or to maintain the state in a desired region. The MPC cost function is based on the sensitivity matrix.

\subsection{Structure for combined control and estimation}

As depicted in Fig. 1, the structure of this closed loop identification algorithm is based on five principal components: a process, a model, an observer, a sensitivity model and a controller.

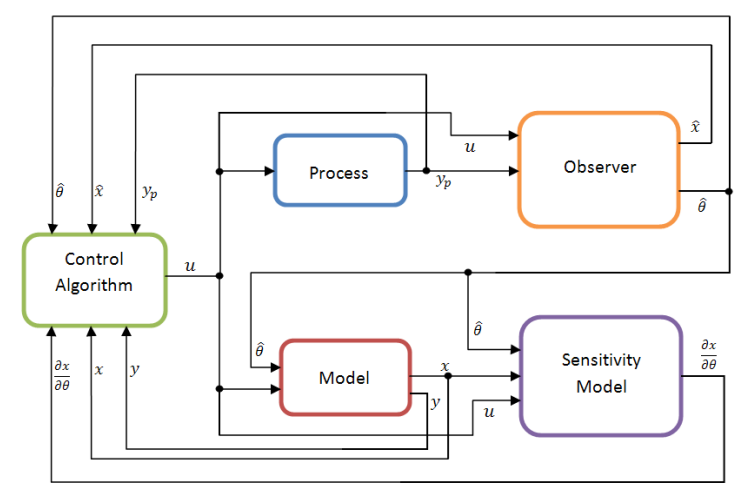

Fig. 1. Proposed closed loop control structure for on-line identification.

\subsection{Control law}

Here, based on the on-line measurements of the process output $y_{p}(k)$, the observer $(O)$, the model $(M)$ and the sensitivity model $\left(M_{\theta}\right)$, the control law is designed to maximize, over the prediction horizon $N_{p}$ and under some input-output constraints, the sensitivity of the model outputs with respect to the model parameters at each current time $k$. First, we define a sensitivity matrix $\bar{y}_{\theta l \mid k}$, which gives at the current instant $k$ the prediction at a future time $l \geq k$ of the normalized outputs sensitivity $y_{\theta}$ as 


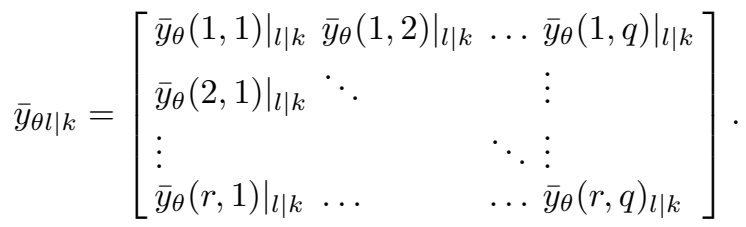

In order to compute this prediction, $\hat{x}(k)$ obtained from the observer is the initial state in $(M)$ at $k . x(t)$ itself is required to solve $x_{\theta}(t)$, while the last estimation $\hat{\theta}(k)$ is also used in both models (it is assumed to be constant over the receding horizon). Then, we can write the FIM as

$$
M_{l \mid k}=\left\|\bar{y}_{\theta l \mid k}\right\|^{2}
$$

which contains the information of the experiment, at the current time $k$, at a future time $l \geq k$. The cost function involved in the MPC strategy is then stated as

$$
\left\{\begin{array}{l}
F\left(\bar{y}_{\theta l \mid k}, u_{l \mid k}, y_{p}(k), \hat{x}_{a}(k)\right)=\frac{1}{N_{p}} \sum_{l=k+1}^{k+N_{p}} M_{l \mid k} \\
u_{l \mid k}=\left\{u(k) \ldots u(l) \ldots u\left(k+N_{p}\right)\right\}, l \in\left[k k+N_{p}\right] .
\end{array}\right.
$$

Then, the E-optimality criterion is defined to maximize the smallest eigenvalue of the matrix $F$. Geometrically, it minimizes the size of the major axis of the join confidence region:

$$
\left\{\begin{array}{l}
u_{l \mid k}^{*}=\arg \max _{u_{|| k}}\left(J\left(u_{l \mid k}\right)=\frac{\lambda_{\min }(F)}{\lambda_{\max }(F)}\right) \\
u_{l \mid k}=\left\{u(k) \ldots u(l) \ldots u\left(k+N_{p}\right)\right\}, l \in\left[k k+N_{p}\right] .
\end{array}\right.
$$

Another interest of a MPC is its ability to handle some constraints:

(1) The inputs must have a physical sense, therefore, they must be bounded in magnitude:

$$
u_{\min } \leq u(k) \leq u_{\max }, \forall k
$$

Velocity constraints may also be added.

(2) The process must be kept into a specified zone. Therefore, constraints on the estimated states and the measured outputs may be build:

$$
g_{\min } \leq g\left(\hat{x}(k), y_{p}(k), u(k)\right) \leq g_{\max }, \forall k
$$

Since the process is open loop unstable, in order to maintain the closed loop behavior in a stable region, we advocate to specify additional output constraints (11). Since these constraints are fictitious, the practical issue is dealing with the design of $g$ and the bounds $g_{\text {min }}$ and $g_{\max }$ : a priori physical knowledge and trial and error with simulations may be used before developing a theoretical proof. However, this method lacks theoretical proof to show that such constrained optimal controller designed for identification purpose renders the closed loop stable, in the sense that the state vector stays within a prescribed (possibly not small) region.

\section{CASE STUDY: ROLLING DELTA WING}

To illustrate the proposed approach, we consider an unstable process which consists of a rolling delta wing system.

\subsection{Step 1: Modelling}

A rolling delta wing is an unstable system where the nonlinear model features a single input $u(t)$, a two component state $x(t)$ and a five unknown constant parameter vector $\theta$ (Jain et al. [2005])

$$
\left\{\begin{aligned}
\dot{x}_{1}(t) & =x_{2}(t) \\
\dot{x}_{2}(t) & =\alpha_{1} \theta_{1} x_{1}(t)+\left(\alpha_{1} \theta_{2}-\alpha_{2}\right) x_{2}(t) \ldots \\
& \ldots+\alpha_{1} \theta_{3} x_{1}^{3}(t) \ldots \\
& \ldots+\alpha_{1} \theta_{4} x_{1}^{2} x_{2}(t) \ldots \\
& \ldots+\alpha_{1} \theta_{5} x_{1} x_{2}^{2}(t) \ldots \\
& \ldots+\alpha_{3} u(t)
\end{aligned}\right.
$$

where $\alpha$ is the known constant parameter vector. The closed loop identification of the vector $\theta$ in this model (12) is discussed in Jain et al. [2005]. A feedback linearizing control is used such that the closed-loop behavior matches with a specified second order linear reference model one with damped sinusoidal input reference. Both designs of this model and its reference input are not really discussed. With their approach, the convergence of the two linear parameter estimations $\left(\theta_{1}\right.$ and $\left.\theta_{2}\right)$ to their target is reported, while the convergence of the three nonlinear parameter estimations $\left(\theta_{3}, \theta_{4}\right.$ and $\left.\theta_{5}\right)$ to their target is not possible.

\subsection{Step 2: Observer design}

Similarly to the work of Jain et al. [2005], both states are here measured on-line. Hence, the purpose of the observer to be designed is to estimate on-line the vector of unknown parameters $\theta_{i}(i=1, \cdots, 5)$. To do so, according to (2), system (12) is augmented by the equation $\dot{\theta}=0$. Consequently, the obtained augmented model is a state affine system up to output nonlinear injection in the following form

$$
\left\{\begin{array}{l}
\dot{x}_{a}(t)=A_{a}(y(t)) x_{a}(t)+B_{a}(u(t)) \\
y(t)=C_{a} x_{a}(t)
\end{array}\right.
$$

where

$$
\begin{aligned}
& x_{a}(t)=\left[\begin{array}{c}
x_{1}(t) \\
x_{2}(t) \\
\theta_{1} \\
\theta_{2} \\
\theta_{3} \\
\theta_{4} \\
\theta_{5}
\end{array}\right] ; B_{a}(u(t))=\left[\begin{array}{c}
0 \\
-\alpha_{3} u(t) \\
0 \\
0 \\
0 \\
0 \\
0
\end{array}\right] ; C_{a}^{T}=\left[\begin{array}{c}
I_{2 \times 2} \\
0_{5 \times 2}
\end{array}\right] ; \\
& A_{a}(y(t))=\left[\begin{array}{ll}
0_{2 \times 1} & A(y(t)) \\
0_{5 \times 1} & 0_{5 \times 6}
\end{array}\right] ; A^{T}(y(t))=\left[\begin{array}{cc}
1 & -\alpha_{2} \\
0 & \alpha_{1} y_{1}(t) \\
0 & \alpha_{1} y_{2}(t) \\
0 & \alpha_{1} y_{1}^{3}(t) \\
0 & \alpha_{1} y_{1}^{2}(t) y_{2}(t) \\
0 & \alpha_{1} y_{1}(t) y_{2}^{2}(t)
\end{array}\right],
\end{aligned}
$$

where $I_{2 \times 2}$ is the $2 \times 2$ identity matrix, $0_{a \times b}$ is the $a \times b$ matrix of zeros. It is clear that system (13) is observable in the sense of the rank condition in the application domain $\left(x_{1} \neq 0\right.$ and $\left.x_{2} \neq 0\right)$. So that, an high gain observer based on the augmented state $x_{a}(t)$ can be designed as follows (see Hammouri and Morales [1990] and Besançon [2007] for more details).

Theorem 1. Assuming that $v(t):=C_{a} \mathcal{X}_{a}\left(t, x_{a}(0)\right)$ is regularly persistent ${ }^{2}$ for

$$
\left\{\begin{array}{l}
\dot{x}_{a}(t)=A_{a}(v(t)) x_{a}(t)+B_{a}(u(t)) \\
y(t)=C_{a} x_{a}(t)
\end{array}\right.
$$

\footnotetext{
2 Regularly persistent inputs for state affine systems are those making the system an LTV system uniformly completely observable in the sense of Kalman.
} 
for any $x_{a}(0)$, then the system admits an exponential observer of the form

$$
\left\{\begin{aligned}
\dot{\hat{x}}_{a}(t) & =A_{a}(y(t)) \hat{x}_{a}(t)+B_{a}(u(t)) \\
& \ldots-R S_{\mu}(t)^{-1} C_{a}^{T}\left(C_{a} \hat{x}_{a}(t)-y_{p}(t)\right) \\
\dot{S}_{\mu}(t) & =-\mu S_{\mu}(t)-A_{a}(y(t))^{T} S_{\mu}(t)-S_{\mu}(t) A_{a}(y(t)) \\
& \ldots+C_{a}^{T} R C_{a}
\end{aligned}\right.
$$

where $S_{\mu}$ is a symmetric positive definite $7 \times 7$ matrix, the positive constant $\mu>0$ and $R>1$ are the observer tuning parameters.

\subsection{Step 3: Sensitivity model}

Based on the model (12), the sensitivity model is simply obtained using the definition (4).

\subsection{Step 4: Control design}

Following section 3, the MPC is designed based on the 2 previous models and the observer. In order to stabilize the closed loop behaviour, and based on the analysis of the simulations of the unstable open-loop behaviour, two additional output constraints (11) are imposed

$$
y_{p 1 \min } \leq y_{p 1}(l) \leq y_{p 1 \max } .
$$

The tuning of the upper and lower bounds in (16) are then tuned by trial and error with simulations: if these two bounds are too close to each other, finding a control value is not easy and does not allow to get sufficiently excited outputs. Hence, parameter estimation is not correct and the constrained optimization problem may not be feasible. On the other side, if these two bounds are too far to each other, closed loop stability is not obtained.

\subsection{Numerical conditions}

The simulation runs are performed under the following conditions where all values are dimensionless ${ }^{3}$

$$
\left\{\begin{array}{l}
\alpha=[0.3540 .0011] \\
\text { input constraints: }-0.01 \leq u(t) \leq 0.01 \\
\text { output constraints: }-0.5 \leq y_{p 1}(t) \leq 0.5 \\
\text { prediction horizon: } N_{p}=5 \\
\text { sampling time: } T_{s}=1 \\
\text { observer tuning parameters: } \mu=0.03 \text { and } R=2 .
\end{array}\right.
$$

Target values and initial estimation errors for $\theta_{i}$ are listed in table 1: in order to see the robustness of the approach, large initial errors in the estimation of $\theta_{i}$ are introduced, even with sign errors. The simulation runs are performed

Table 1. $\theta$ : Target values and initial estimation errors.

\begin{tabular}{cccccc}
\hline$\theta$ & 1 & 2 & 3 & 4 & 5 \\
\hline $\begin{array}{c}\text { Target } \\
\text { (absolute } \\
\text { values) }\end{array}$ & -0.05686 & 0.03254 & 0.07334 & -0.3597 & 1.46681 \\
\hline $\begin{array}{c}\text { Initial } \\
\text { error } \\
(\%)\end{array}$ & 80 & -200 & 200 & 80 & -200 \\
\hline
\end{tabular}

based on our odoe4ope ${ }^{4}$ software based on fmincon in Matlab.

3 The time, the input and the states are also dimensionless.

4 To use this software, please visit http://odoe4ope.univ-lyon1.fr

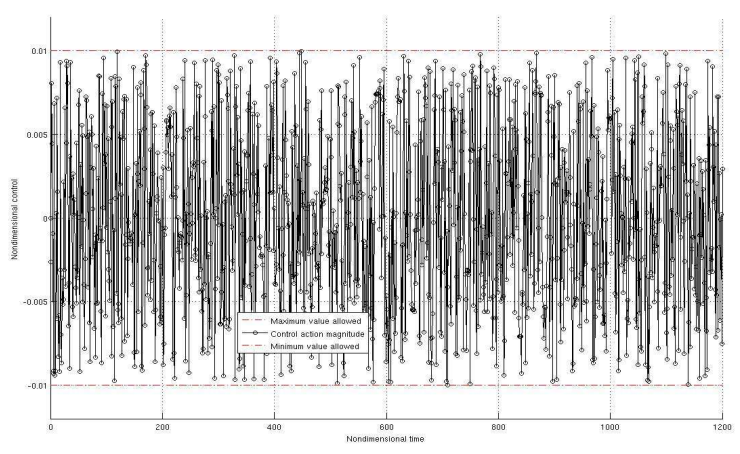

Fig. 2. Closed loop optimal input.
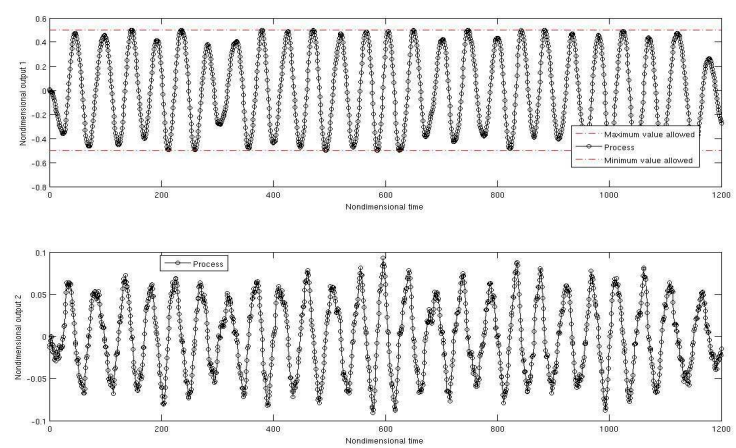

Fig. 3. Closed loop outputs (top: $y_{p 1}$, bottom: $y_{p 2}$ ).
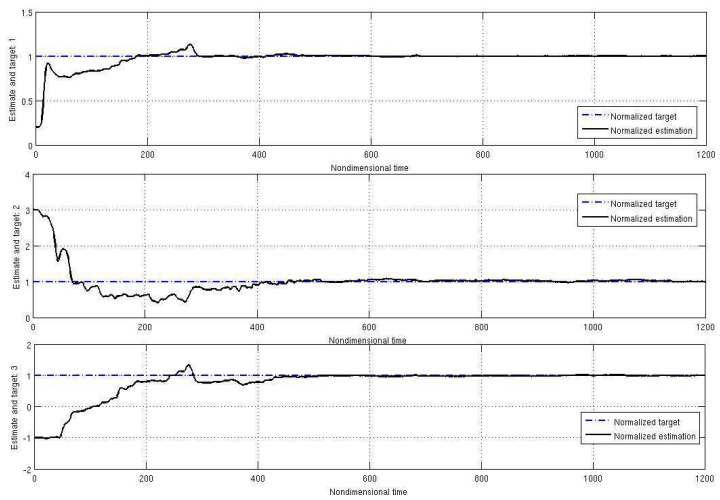

Fig. 4. Closed loop estimation (from the top to the bottom: 3 first model parameters).

\subsection{Numerical results and analysis}

As it can be seen, both objectives are fulfilled: the closed loop control determined (Fig. 2) allows to stabilize the rolling delta wing behaviour (see both outputs in Fig. 3) while all 5 parameters $\theta_{i}$ almost reach their target values between 300 and 600: in Fig. 4 and Fig. 5, the five target parameters and their estimations are normalized (hence, 1 is the target). Based on the mean value of the 60 last time values, the final error of estimation of the $\theta_{i}$ has decreased (see table 2) from initially few tens or few hundreds $\%$ to about $1 \%$. Hence, all model parameters are finally estimated with a high accuracy. 


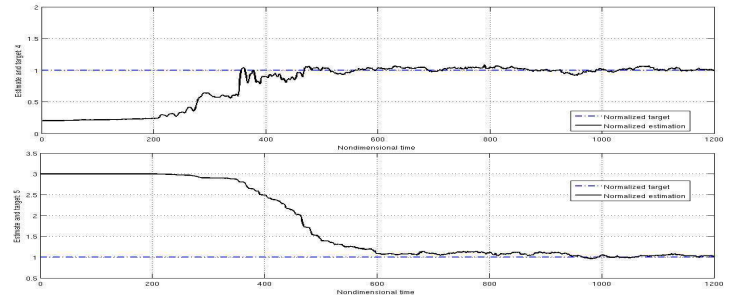

Fig. 5. Closed loop estimation (from the top to the bottom: 2 last model parameters)

Table 2. $\theta$ : Final estimation error.

\begin{tabular}{cccccc}
\hline$\theta$ & 1 & 2 & 3 & 4 & 5 \\
\hline $\begin{array}{c}\text { Final } \\
\text { error } \\
(\%)\end{array}$ & 0.1 & 0.4 & 0.8 & -0.26 & -2.88 \\
\hline
\end{tabular}

\section{CONCLUSION}

This paper presents a new approach for the on-line closed loop identification of unknown parameters of nonlinear multivariable systems (in terms of state dynamics and/or in terms of parameters) in one experiment. Based on the observer design theory, on the sensitivity model and on the predictive control approach, a closed-loop optimal experiment design has been developed. The efficiency of the proposed algorithm has been demonstrated in a stepby-step description for a open loop unstable delta wing process. To guaranty the stability of the closed loop, output constraints were imposed, where the bounds are for the moment designed by trial and error. The correct estimation of all five constant model parameters and the closed loop stabilization has been shown. Compared to a previous work, this method helps to estimate with a very good accuracy all initially unknown model parameters. In future works, we will provide the mathematical conditions for the method which guaranty the stability of the closed loop by carrying out a Lyapunov analysis. Also, the cases where the process state is not fully measured or where the model parameters are time-varying will be tackled.

\section{REFERENCES}

Besançon, G. (2007). Identification of parametric models from experimental data. Lecture Notes in Control and Information Sciences 363, Springer-Verlag, Berlin Heidelberg.

Biagiola, S. and Figueroa, J. (2004). Application of state estimation based NMPC to an unstable nonlinear processes. Chemical Engineering Science, (59), 46014612.

Boizot, N., Busvelle, E., and Gauthier, J.P. (2010). An adaptive high-gain observer for nonlinear systems. Automatica, 46, 1483-1488.

Flila, S., Dufour, P., and Hammouri, H. (2008). Optimal input design for on-line identification: a coupled observer-MPC approach. In Proceedings of the 17th IFAC World Congress, 11457-11462. Seoul, South Korea.

Forssell, U. and Ljung, L. (1998). Identification of unstable systems using output error and box-jenkins model structures. In Proceedings of the 29th IEEE CSS Chinese Control Conference, 3932-3937.
Franceschini, G. and Macchietto, S. (2008). Optimal experiment design for linear systems with input-output constraints. State of the art. Chemical Engineering Science, 63, 4846-4872.

Genceli, H. and Nikolaou, M. (1996). New approach to constrained predictive control with simultaneous model identification. AIChE Journal, 42(10), 857-2868.

Goodwin, G. and Payne, R. (1979). Dynamic system identification: Experiment design and data analysis. Academic Press, New York.

Hammouri, H. and Morales, J.D.L. (1990). Observer synthesis for state-affine systems. 29th IEEE Conf. on Decision and Control, (3), 784-785.

Heidarinejad, M., Liu, J., and Christofides, P. (2013). Algorithms for improved fixed-time performance of Lyapunov-based economic model predictive control of nonlinear systems. Journal of Process Control, 23, 404414.

Jain, H., Kaul, V., and Ananthkrishnan, N. (2005). Parameter estimation of unstable, limit cycling systems using adaptive feedback linearization: example of delta wing roll dynamics. Journal of Sound and Vibration, 939-960.

Jayasankar, B., Huang, B., and Ben-Zv, A. (2010). Receding horizon experiment design with application in sofc parameter estimation. In Proceedings of the International Symposium on Dynamics and Control of Process Systems, 527-532. Leuven, Belgium.

Ma, J., Qin, J., Salsbury, T., and Xu, P. (2012). Demand reduction in building energy systems based on economic model predictive control. Chemical Engineering Science, 67, 92-100.

Mehra, R. (1974). Optimal input signals for parameter estimation in dynamic systems survey and new results. IEEE Transactions on Automatic Control, AC-19, 753768.

Nadri, M., Hammouri, H., and Astorga, C. (2004). Observer design for continuous discrete-time state affine systems up to output injection. European Journal of Control, 10(3), 252-262.

Nadri, M., Hammouri, H., and Grajales, R. (2013). Observer design for uniformly observable systems with sampled measurements. IEEE Transactions on Automatic Control, 58(3), 757-762.

Rawlings, J., Angeli, D., and Bates, C. (2012). Fundamentals of economic model predictive control. In Proceedings of the 51st IEEE Conference on Decision and Control, 3851-3861. Maui, Hawaii, USA.

Richalet, J. and O'Donovan, D. (2009). Predictive Functional Control. Springer-Verlag London Limited.

Zarrop, M. (1979). Optimal experiment design for dynamic system identification, volume 21. Lecture Notes in Control and Information Sciences, Springer Verlag,Berlin, New York.

Zhou, J., Aamo, O., and Kaasa, G. (2009). Adaptive observer design for nonlinear systems with parametrice uncertainties in unmeasured state dynamics. In Proceedings of the 48th IEEE Conference on Decision and Control and 28th Chinese Control Conference, 44144419. Shanghai, China.

Zhu, Y. and Huang, B. (2011). Constrained recedinghorizon experiment design and parameter estimation in the presence of poor initial conditions. AIChE Journal, 57(10), 2808-2820. 